\title{
Predictions for nuclear structure functions from the impact-parameter-dependent Balitsky-Kovchegov equation
}

\author{
J. Cepila $\odot$, J. G. Contreras, and M. Matas $\odot$ \\ Faculty of Nuclear Sciences and Physical Engineering, Czech Technical University in Prague, 11000 Prague, Czech Republic
}

(Received 26 February 2020; revised 24 August 2020; accepted 28 September 2020; published 16 October 2020)

\begin{abstract}
In this work we present dipole scattering amplitudes, including the dependence on the impact-parameter, for a variety of nuclear targets of interest for the electron-ion colliders (EICs) being currently designed. These amplitudes are obtained by numerically solving the Balitsky-Kovchegov equation with the collinearly improved kernel. Two different cases are studied: initial conditions representing the nucleus under consideration and the solutions based on an initial condition representing a proton complemented by a Glauber-Gribov prescription to obtain dipole-nucleus amplitudes. We find that the energy evolution of these two approaches differ. We use the obtained dipole scattering amplitudes to predict (i) nuclear structure functions that can be measured in deepinelastic scattering at EICs and (ii) nuclear suppression factors that reveal the energy evolution of shadowing for the different cases we studied. We compare our predictions with the available data.
\end{abstract}

DOI: 10.1103/PhysRevC.102.044318

\section{INTRODUCTION}

Feasibility studies for electron-ion colliders (EICs), like those proposed in the USA [1] or at CERN [2], are an essential ingredient towards the design of these machines. Inclusive measurements are among the most important observables in this context. In particular, the study of the structure function $F_{2}^{A}\left(x, Q^{2}\right)$ at small Bjorken $x$ for photons of virtuality $Q^{2}$ at a perturbative scale, and for a variety of nuclei $A$, is expected to yield a new understanding of the high-energy limit of quantum chromodynamics (QCD). Comparison of these measurements with those reported by $\mathrm{H} 1$ and ZEUS [3] for the corresponding structure function of the proton, $F_{2}^{p}\left(x, Q^{2}\right)$ promise to shed new light on the origin of shadowing, the phenomenon that the parton distributions of nucleons bound in a nucleus are suppressed with respect to those of free nucleons [4].

At small values of $x$, the dominant parton distribution is that of gluons; thus the case of gluon shadowing has been the focus of attention for theorists since a long time; see, e.g., Refs. [5,6]. A process expected to occur in this kinematic regime is saturation, namely, the fact that the density of gluons is so high that they start to interact with each other, even in the domain of perturbative QCD (for a recent review, see Ref. [7]). An early equation to describe saturation was introduced in the seminal work [8], while nowadays it is common to use the Balitsky-Kovchegov (BK) equation for this type of studies. The leading-order BK equation, discussed in Sec II, was derived in Refs. $[9,10]$ using two independent approaches. Later

Published by the American Physical Society under the terms of the Creative Commons Attribution 4.0 International license. Further distribution of this work must maintain attribution to the author(s) and the published article's title, journal citation, and DOI. Funded by $S C O A P^{3}$. on, corrections to account for the running of the coupling $[11,12]$ as well as the resummation of other logarithmic contributions $[13,14]$ were incorporated into this formalism. In the approximation of considering a large homogeneous target; that is, disregarding the impact-parameter dependence, this equation has been successfully used to describe the existing $F_{2}^{p}\left(x, Q^{2}\right)$ data, see e.g., Refs. [14,15].

The first attempt at solving the BK equation including the impact-parameter dependence [16] found that the solutions developed so-called Coulomb tails: an unphysical growth of the amplitude at large impact parameters. Nonetheless, using some extra corrections it was possible to describe the structure-function data of the proton [17,18]. Recently, our group discovered that, using the collinearly improved kernel introduced in Ref. [14], the problem of Coulomb tails is tamed such that a successful phenomenology using the BK equation is possible $[19,20]$. In a further development, it was suggested to change the evolution variable in the BK equation [21]. In Ref. [22] it was found that the new and the old approaches yield the same phenomenology at next to leading order (NLO) for the structure functions measured at the Hadron-Electron Ring Accelerator (HERA) at DESY, so in this work we will used the more traditional approach and explore the new proposal somewhere else.

In this article, we solve the BK equation with the collinearly improved kernel for different nuclei of importance for future EICs and predict their structure functions as well as the corresponding nuclear suppression factors, which are a direct measurement of shadowing. We study two cases: solutions obtained from an initial condition representing the nuclei (denoted as b-BK-A below), and solutions of the proton case coupled to a Glauber-Gribov prescription to obtain the nuclear structure functions (denoted as b-BK-GG). Other approaches to the computation of nuclear structure functions can be found, for example, in Refs. [23-27]. 
The rest of the text is organized as follows: Sec. II contains a brief review of the formalism, including the definition of the initial conditions and the values of the parameters used in the computation. Section III discusses the behavior of the dipole scattering amplitudes obtained by solving the BK equation for the different nuclei and the b-BK-A and b-BK-GG approaches. Section IV presents our predictions for the nuclear structure functions and nuclear suppression factors for all cases under study as well as a comparison with the available data. Finally, in Sec. V we provide a brief summary of the presented work as well as an outlook of future steps.

\section{FORMALISM}

\section{A. The Balitsky-Kovchegov equation with the collinearly improved kernel}

The leading-order Balitsky-Kovchegov equation $[9,10]$ is

$$
\begin{aligned}
\frac{\partial N(\vec{r}, \vec{b}, Y)}{\partial Y}= & \int d \vec{r}_{1} K\left(r, r_{1}, r_{2}\right)\left[N\left(\vec{r}_{1}, \overrightarrow{b_{1}}, Y\right)+N\left(\vec{r}_{2}, \overrightarrow{b_{2}}, Y\right)\right. \\
& \left.-N(\vec{r}, \vec{b}, Y)-N\left(\vec{r}_{1}, \vec{b}_{1}, Y\right) N\left(\overrightarrow{r_{2}}, \overrightarrow{b_{2}}, Y\right)\right] .
\end{aligned}
$$

It describes the evolution in rapidity $Y$ of the dipole scattering amplitude $N(\vec{r}, \vec{b}, Y)$. Here, the sizes of the mother and daughter dipoles are $r \equiv|\vec{r}|, r_{1} \equiv\left|\overrightarrow{r_{1}}\right|$, and $r_{2} \equiv\left|\overrightarrow{r_{2}}\right| \equiv$ $\left|\vec{r}-\vec{r}_{1}\right|$, respectively. The magnitudes of the corresponding impact parameters between these dipoles and the hadronic target are $b \equiv|\vec{b}|, b_{1} \equiv\left|\overrightarrow{b_{1}}\right|, b_{2} \equiv\left|\overrightarrow{b_{2}}\right|$. All these vectors are two dimensional and live in the impact-parameter plane. The impact-parameter vectors of the daughter dipoles are given by

$$
b_{1}=\left|\vec{b}+\frac{\overrightarrow{r_{2}}}{2}\right|, \quad b_{2}=\left|\vec{b}-\frac{\vec{r}_{1}}{2}\right|
$$

The dipole scattering amplitude in Eq. (1) depends on the size of the dipole $(r)$, the magnitude of the impact parameter $(b)$, the orientation of the impact parameter $\left(\phi_{b}\right)$, and the angle between $\vec{r}$ and $\vec{b}\left(\theta_{r b}\right)$. We solve the BK equation for a rotationally symmetric target so there is no dependence on $\phi_{b}$ and this property is preserved by the evolution. Furthermore, to reduce the degrees of freedom, at each step of the evolution we fix $\theta_{r b}=0$. (We have checked that fixing this angle to other values produces similar results.) Under these conditions, Eq. (1) reduces to

$$
\begin{aligned}
\frac{\partial N(r, b, Y)}{\partial Y}= & \int d \vec{r}_{1} K\left(r, r_{1}, r_{2}\right)\left[N\left(r_{1}, b_{1}, Y\right)+N\left(r_{2}, b_{2}, Y\right)\right. \\
& \left.-N(r, b, Y)-N\left(r_{1}, b_{1}, Y\right) N\left(r_{2}, b_{2}, Y\right)\right] . \quad \text { (3) }
\end{aligned}
$$

For the kernel we use the recently proposed collinearly improved version [13]

$K_{\mathrm{ci}}\left(r, r_{1}, r_{2}\right)=\frac{\bar{\alpha}_{s}}{2 \pi} \frac{r^{2}}{r_{1}^{2} r_{2}^{2}}\left[\frac{r^{2}}{\min \left(r_{1}^{2}, r_{2}^{2}\right)}\right]^{ \pm \bar{\alpha}_{s} A_{1}} K_{\mathrm{DLA}}\left(\sqrt{L_{r_{1} r} L_{r_{2} r}}\right)$,

where (see also Ref. [28])

$$
K_{\mathrm{DLA}}(\rho)=\frac{J_{1}\left(2 \sqrt{\bar{\alpha}_{s} \rho^{2}}\right)}{\sqrt{\overline{\bar{\alpha}}_{s} \rho}}
$$

TABLE I. Values of the parameters of the Woods-Saxon distribution, see Eq. (8), used in the computations reported in this text, and the value of the $Q_{s 0}^{2}(A)$ parameter obtained as explained in the text. The Woods-Saxon parameters are taken from Ref. [31].

\begin{tabular}{cclclc}
\hline \hline Nucleus & $A$ & $R(\mathrm{fm})$ & $a(\mathrm{fm})$ & $\rho_{0}\left(\mathrm{fm}^{-3}\right)$ & $Q_{s 0}^{2}(A)\left(\mathrm{GeV}^{2}\right)$ \\
\hline $\mathrm{Al}$ & 27 & 2.84 & 0.569 & 0.2015 & 0.315 \\
$\mathrm{Ca}$ & 40 & 3.51 & 0.563 & 0.17611 & 0.341 \\
$\mathrm{Fe}$ & 56 & 3.980 & 0.569 & 0.17655 & 0.390 \\
$\mathrm{Cu}$ & 64 & 4.2 & 0.569 & 0.1746 & 0.409 \\
$\mathrm{~W}$ & 184 & 6.510 & 0.535 & 0.1493 & 0.553 \\
$\mathrm{~Pb}$ & 208 & 6.624 & 0.549 & 0.16 & 0.609 \\
\hline \hline
\end{tabular}

$J_{1}$ is the Bessel function, the anomalous dimension is $A_{1}=$ $11 / 12$, and $L_{r_{i} r}=\ln \left(r_{i}^{2} / r^{2}\right)$. The sign is positive when the size of the original dipole is smaller than the size of each of the daughter dipoles and negative otherwise. The smallest dipole prescription is used for the running coupling: $\bar{\alpha}_{s}=$ $\alpha_{s}\left(r_{\min }\right) N_{c} / \pi$, where $r_{\min }=\min \left(r_{1}, r_{2}, r\right)$. Note that this prescription has also been put forward as the natural scale for the BK equation at next-to-leading order [29]. The variablenumber-of-flavors scheme is used with the same parameters as in our previous work [19,20].

\section{B. Glauber-Gribov approach to the nuclear dipole amplitude}

Following Ref. [23], one can use the solution of the BK equation for the case of a proton target to obtain the dipole scattering amplitude for a nuclear target by using a GlauberGribov approach

$$
N^{A}(r, b, Y)=\left[1-\exp \left(-\frac{1}{2} T_{A}(b) \sigma_{q \bar{q}}(Y, r)\right)\right],
$$

with

$$
\sigma_{q \bar{q}}(Y, r)=\int d^{2} \vec{b} 2 N^{p}(r, b, Y)
$$

This approach has been used in other studies, e.g., those reported in Refs. [23,24] (see also Ref. [26] for a more general approach that reduces to the Glauber-Gribov case for large nuclei). The nuclear thickness function $T_{A}(b)$ is obtained from a Woods-Saxon distribution for the nuclear matter density

$$
\rho_{A}(x, y, z)=\rho_{0} \frac{1}{\exp [(r-\mathrm{R}) / \mathrm{a}]+1}
$$

[where $r \equiv\left(x^{2}+y^{2}+z^{2}\right)^{1 / 2}$ ], by integrating it over the longitudinal coordinate $z$

$$
T_{A}(b)=\int_{-\infty}^{+\infty} d z \rho_{A}(x, y, z)
$$

with the $x$ and $y$ coordinates in the impact-parameter plane. It is normalized according to $\int d^{2} \vec{b} T_{A}(b)=A$. (See, for example, Ref. [30] for full details on the formalism.) The values of the Woods-Saxon parameters are given in Table I. This approach is denoted as b-BK-GG in what follows. 


\section{Initial conditions for the nuclear targets}

To solve the BK equation, an initial condition is needed. In our previous work $[19,20]$, we introduced a new functional form for the initial condition given by

$$
N^{p}(r, b, Y=0)=1-\exp \left(-\frac{1}{2} \frac{Q_{s 0}^{2}}{4} r^{2} T_{p}\left(b_{q_{1}}, b_{q_{2}}\right)\right),
$$

where $Q_{s 0}^{2}$ is a free parameter representing the saturation scale at zero impact parameter, and $b_{q_{i}}$ are the impact parameters of the quark and antiquark forming the dipole.

For the case of the proton, we assumed a Gaussian-like distribution which leads to

$$
T_{p}\left(b_{q_{1}}, b_{q_{2}}\right)=\left[\exp \left(-\frac{b_{q_{1}}^{2}}{2 B_{G}}\right)+\exp \left(-\frac{b_{q_{2}}^{2}}{2 B_{G}}\right)\right] .
$$

The parameter $B_{G}$ was set to $3.2258 \mathrm{GeV}^{-2}$, while $Q_{s 0}^{2}$ took the value $0.496 \mathrm{GeV}^{2}$. With these values, a satisfactory description of HERA and LHC data on the proton structure functions and exclusive production of vector mesons is achieved [19,20].

Here, we follow a similar approach for the nuclear case, but assuming a Woods-Saxon instead of a Gaussian distribution:

$$
N^{A}(r, b, Y=0)=1-\exp \left(-\frac{1}{2} \frac{Q_{s 0}^{2}(A)}{4} r^{2} T_{A}\left(b_{q_{1}}, b_{q_{2}}\right)\right)
$$

with

$$
T_{A}\left(b_{q_{1}}, b_{q_{2}}\right)=k\left[T_{A}\left(b_{q_{1}}\right)+T_{A}\left(b_{q_{2}}\right)\right],
$$

where the factor $k$ ensures that $k T_{A}(0)=1$. This approach is denoted as b-BK-A in what follows.

As the nuclear parameters are already fixed, the only free parameter is $Q_{s 0}^{2}(A)$. We have fixed these parameters by using $N(r, b, Y=0)$ where $Y=\ln \left(x_{0} / x\right)$ with $x_{0} \equiv 0.008$. This dipole scattering amplitude at the initial rapidity is used to compute structure functions and to compare them with the predictions obtained using the EPPS16 nuclear parton distributions [32].

In detail, we have varied the value of the $Q_{s 0}^{2}(A)$ parameter in order to get a small relative deviation from the structure function $F_{2}\left(x=0.008, Q^{2}\right)$ as predicted by the EPPS 16 parton distribution functions (PDFs). The comparison is done for the following values of the photon virtuality: $Q^{2} \in[3.5$, $4.5,6.5,8.5,10,12,15,18,22,27] \mathrm{GeV}^{2}$ to avoid the nonperturbative region at very low $Q^{2}$ and to stay in the region of virtualities where the $\mathrm{BK}$ equation is expected to work the best. We have used LHAPDFs [33] to obtain the PDF sets and the APFEL software [34,35] for the computation of the structure function. The values obtained for $Q_{s 0}^{2}(A)$ by this procedure are reported in the last column of Table I.

Interestingly, this parameter follows a linear behavior as a function of $A^{1 / 3}$, as shown in Fig. 1. This opens the possibility of studying other nuclei for which there is currently no information in the EPPS16 set of parton distributions.

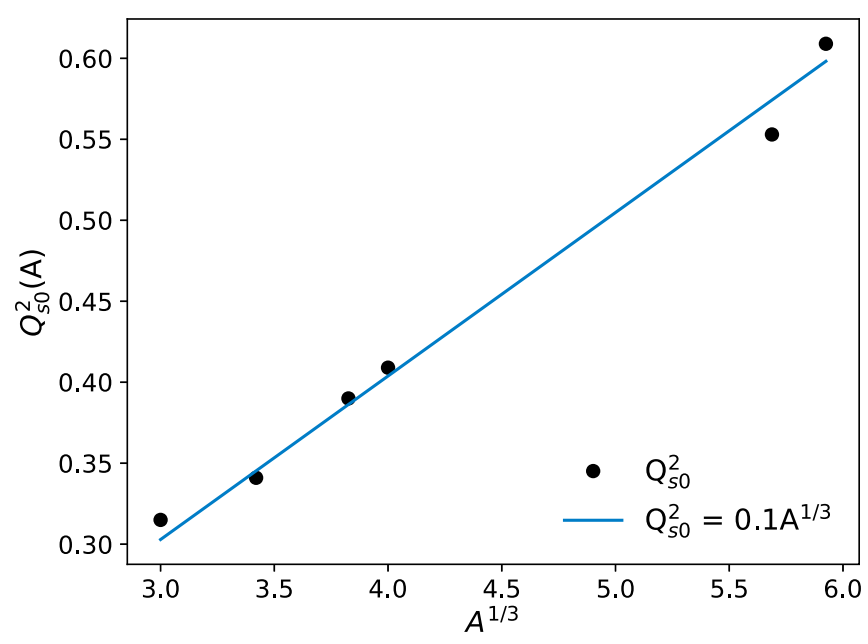

FIG. 1. Dependence of the $Q_{s 0}^{2}(A)$ parameter as a function of $A^{1 / 3}$ (solid bullets) compared with a linear function (blue line). See text for details.

\section{BEHAVIOR OF THE DIPOLE SCATTERING AMPLITUDE}

The dipole scattering amplitude in the b-BK-A approach computed using the colinearly improved kernel with the initial condition given by Eq. (12) is shown in Fig. 2 for two values of the rapidity and at a fixed value of $r$, respectively $b$, for three different nuclei. The chosen rapidity values are the initial condition $(Y=0)$ and $Y=5$, which corresponds to $x \approx 5.4 \times 10^{-5}$ representing the case of a dipole scattering amplitude evolved to a rapidity of potential interest of future EICs. The impact-parameter dependence is clearly different for the three depicted nuclei, reflecting their different sizes, while the shape of the amplitude as a function of $r$ is similar for the three cases. The main effects of the evolution are the growth of the profile in impact parameter, the softening of the large $r$ behavior, and a small advancement of the wavefront towards smaller dipoles.

Figure 3 shows a comparison of the two methods, b-BK-A and b-BK-GG, to compute the nuclear dipole scattering amplitude presented above. The differences are remarkable. The absolute value of the amplitude for dipoles of size $1 / \mathrm{GeV}$ is substantially smaller for b-BK-A. Regarding the dependence on the dipole size at an impact parameter of $0.1 / \mathrm{GeV}$, the behavior of the b-BK-A and b-BK-GG models is different. The evolved amplitude for the b-BK-A case decays at large sizes, while for the b-BK-GG case it remains flat in this region. The reason of the b-BK-GG behavior is that the small growth of the amplitude at large values of $r$ gets a large weight when integrating the proton amplitude over impact parameter in Eq. (7), and this weight gets exponentiated when the Glauber-Gribov prescription is applied in Eq. (6).

The fact that very large dipole sizes contribute to the dipole scattering amplitude is not ideal. Such large dipole sizes are in the nonperturbative region. Such a behavior has been observed in all studies that considered only the dependence of the amplitude on the dipole size: $N=N(r ; Y)$. In the case of the b-BK-A approach, and as already observed for the case 

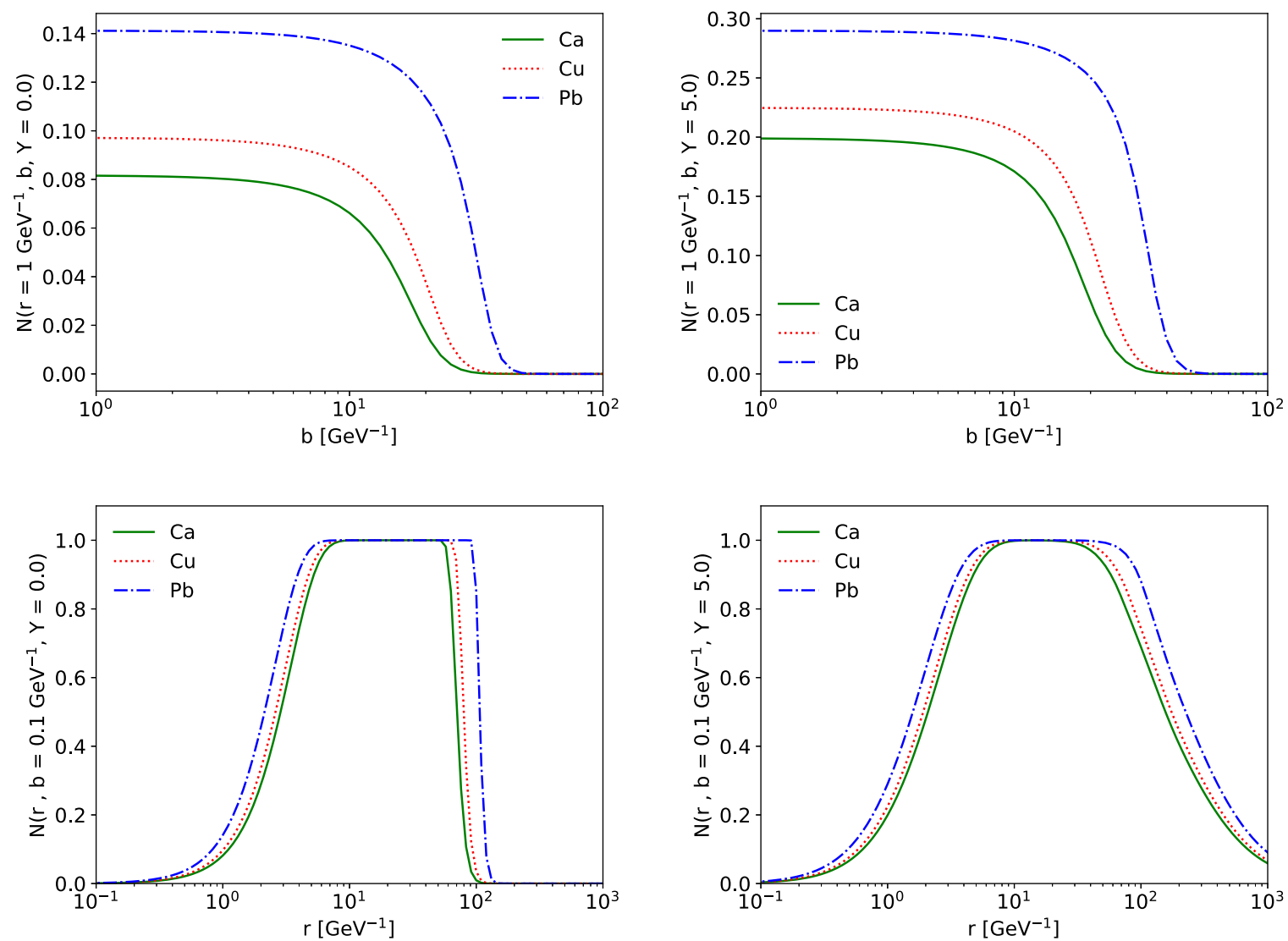

FIG. 2. Dipole scattering amplitude in the b-BK-A approach at the initial condition (left) and after evolution to $Y=5$ (right) as a function of the impact parameter for a dipole size $r=1 / \mathrm{GeV}$ (upper panels), and as a function of the dipole size for an impact parameter $b=0.1 / \mathrm{GeV}$ (lower panels).

of the proton in Ref. [20], the situation is much improved and very large dipoles are suppressed. Note that these large dipoles do not contribute to the phenomenological applications discussed below, because the dipole scattering amplitude is convoluted with a wave function that selects the contribution of dipoles with sizes in the perturbative region.

These differences between the dipole scattering amplitudes in the two approaches reflect themselves in one of the most important parameters that can be obtained from these objects: the saturation scale and its evolution. As is standard, we define the saturation scale at a given rapidity and a fixed impact parameter as the dipole size that produces a scattering amplitude equal to a constant that commonly is chosen to be one half. Figure 4 shows the behavior of the saturation scale at an impact parameter of $0.01 / \mathrm{GeV}$ for two rapidities as a function of $A^{1 / 3}$. The saturation scale shows a linear behavior in this representation. The intercept is larger for b-BK-A with respect to b-BK-GG, while the slope is smaller. The evolution of both the intercept and the slope seems to be different in both cases. For all values of $A$ the saturation scale at $Y=5$ is smaller for b-BK-A than for b-BK-GG predictions. Note that the figure would look the same at other values of the impact parameter due to the flat form of the dipole scattering amplitude as shown in Fig. 3. Only for larger values of the impact parameter, around 4 to $5 \mathrm{GeV}^{-1}$, the drop at the border of the nuclei changes the behavior of Fig. 4 .

\section{PREDICTIONS FOR NUCLEAR STRUCTURE FUNCTIONS AND NUCLEAR SUPPRESSION FACTORS}

\section{A. Relation between the dipole scattering amplitude and the structure function}

Using as input the dipole scattering amplitudes, the structure function $F_{2}^{A}\left(x, Q^{2}\right)$ is computed as

$$
\begin{aligned}
& F_{2}^{A}\left(x, Q^{2}\right) \\
& \quad=\frac{Q^{2}}{4 \pi^{2} \alpha_{\mathrm{em}}} \int \sum_{i} d \vec{r} d \vec{b} d z\left|\Psi_{T, L}^{i}(z, \vec{r})\right|^{2} \frac{d \sigma^{q \bar{q}}(\vec{r}, \tilde{x})}{d \vec{b}},
\end{aligned}
$$

where, following Ref. [36], $\tilde{x}=x\left[1+\left(4 m_{q_{i}}^{2}\right) / Q^{2}\right]$ with $m_{q_{i}}$ being the mass of the $i$ quark. The dipole-target cross section is related to the dipole scattering amplitude by

$$
\frac{d \sigma^{q \bar{q}}(\vec{r}, x)}{d \vec{b}}=2 N^{A}(\vec{r}, \vec{b}, x) .
$$

Finally, the wave function representing the probability of a virtual photon splitting into a quark-antiquark dipole, and following the notation of Ref. [36], is

$$
\begin{aligned}
\left|\Psi_{T}^{i}\left(z, \vec{r}, Q^{2}\right)\right|^{2}= & \frac{3 \alpha_{\mathrm{em}}}{2 \pi^{2}} e_{q_{i}}^{2}\left\{\left[z^{2}+(1-z)^{2}\right] \epsilon^{2} K_{1}^{2}(\epsilon r)\right. \\
& \left.+m_{q_{i}}^{2} K_{0}^{2}(\epsilon r)\right\},
\end{aligned}
$$



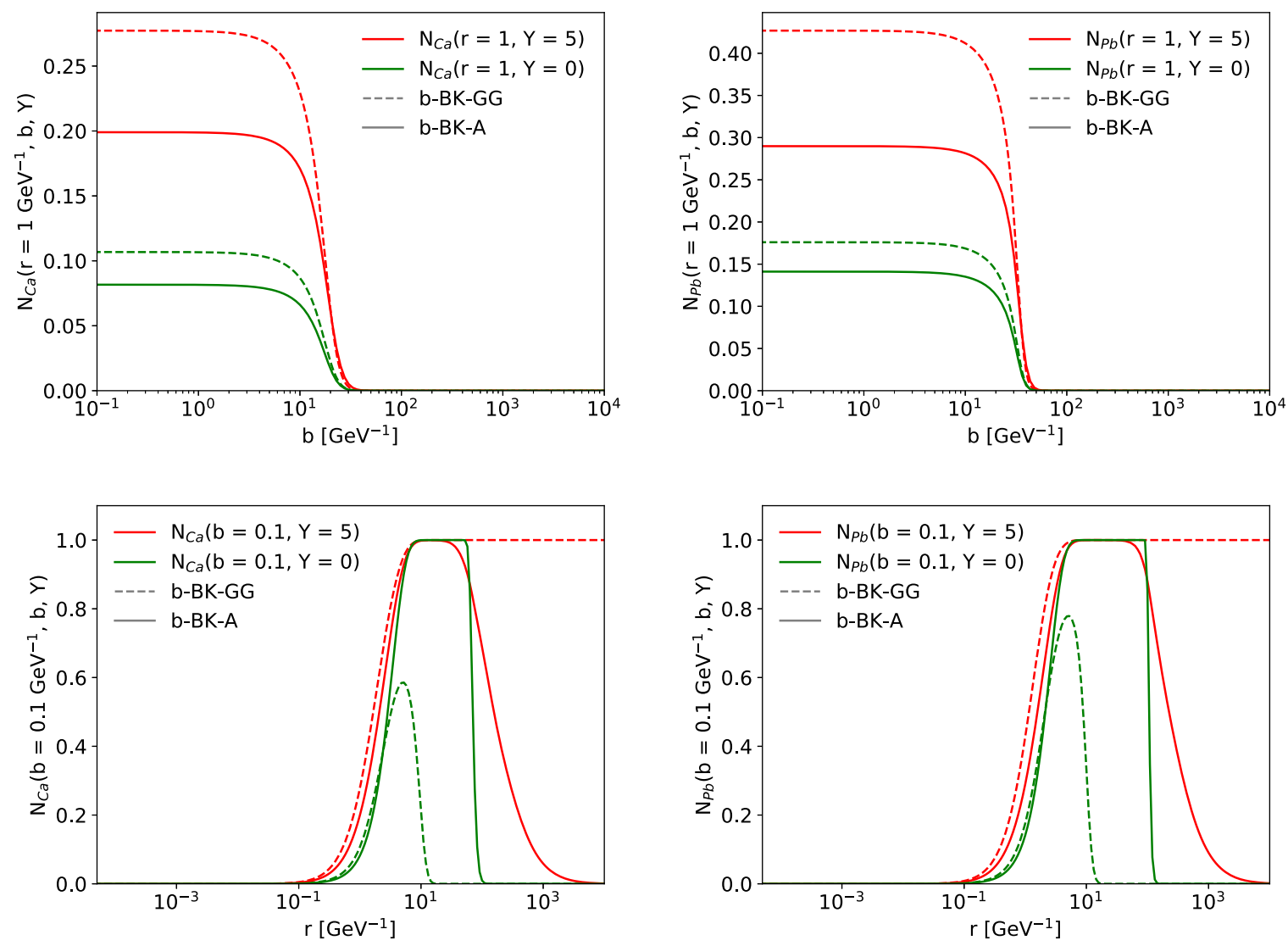

FIG. 3. Comparison of the dipole scattering amplitudes computed for b-BK-A (solid line) with the b-BK-GG approach (dashed line). The comparisons are done at $Y=0$ and $Y=5$ for two nuclei, $\mathrm{Ca}$ (left) and $\mathrm{Pb}$ (right) as a function of the impact parameter for a dipole size $r=1 / \mathrm{GeV}$ (upper panels), and as a function of the dipole size for an impact parameter $b=0.1 / \mathrm{GeV}$ (lower panels).

and

$$
\left|\Psi_{L}^{i}\left(z, \vec{r}, Q^{2}\right)\right|^{2}=\frac{3 \alpha_{\mathrm{em}}}{2 \pi^{2}} e_{q_{i}}^{2}\left[4 Q^{2} z^{2}(1-z)^{2} K_{0}^{2}(\epsilon r)\right]
$$

for the transverse and longitudinal polarization of the incoming photon, respectively. The total wave function is

$$
\left|\Psi_{T, L}^{i}(z, \vec{r})\right|^{2}=\left|\Psi_{T}^{i}(z, \vec{r})\right|^{2}+\left|\Psi_{L}^{i}(z, \vec{r})\right|^{2} .
$$

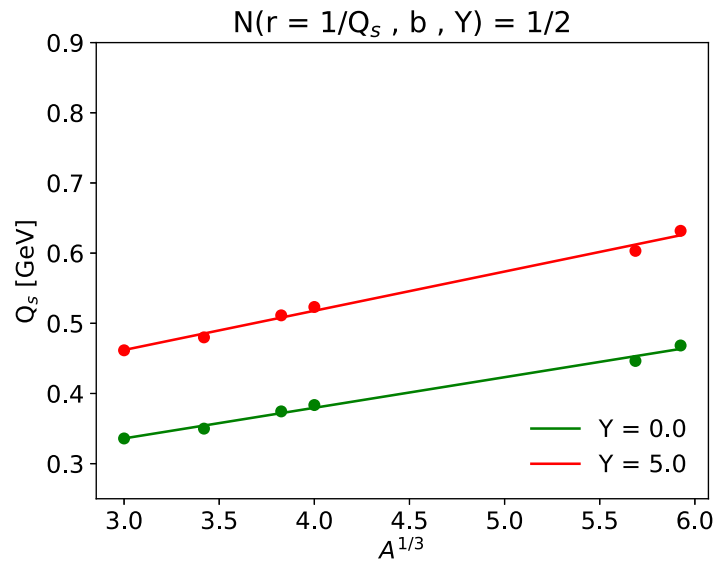

In these equations, $K_{0}$ and $K_{1}$ are the MacDonald functions, $z$ is the fraction of the total longitudinal momentum of the photon carried by the quark, $e_{q_{i}}$ is the fractional charge (in units of elementary charge) of quark $i, \alpha_{\mathrm{em}}=1 / 137$ and $\epsilon^{2}=$ $z(1-z) Q^{2}+m_{q_{i}}^{2}$. As in our previous work $[19,20]$ we set the quark masses to $100 \mathrm{MeV} / c^{2}$ for light, $1.3 \mathrm{GeV} / c^{2}$ for charm, and $4.5 \mathrm{GeV} / c^{2}$ for bottom quark. As reported, for example,

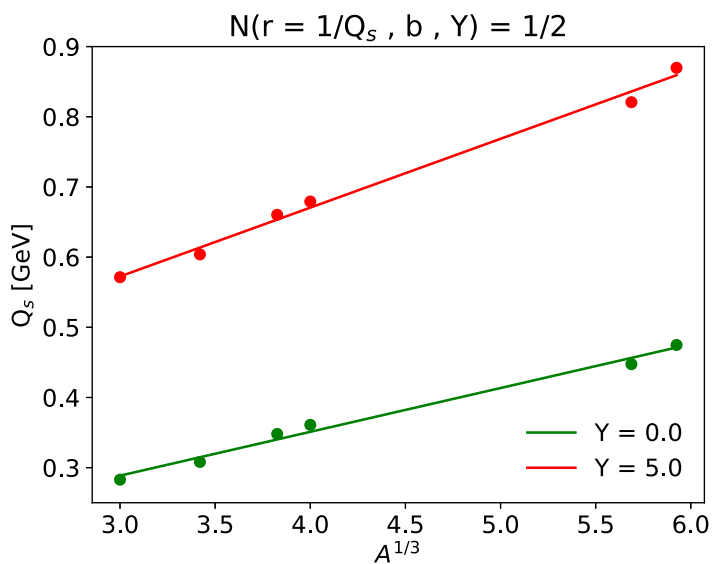

FIG. 4. Saturation scale at two different rapidities for an impact parameter of $0.01 / \mathrm{GeV}$ for the b-BK-A (left) and b-BK-GG (right) approaches. The solid bullets are the results from the evolution and are well described by a linear function. See text for details. 

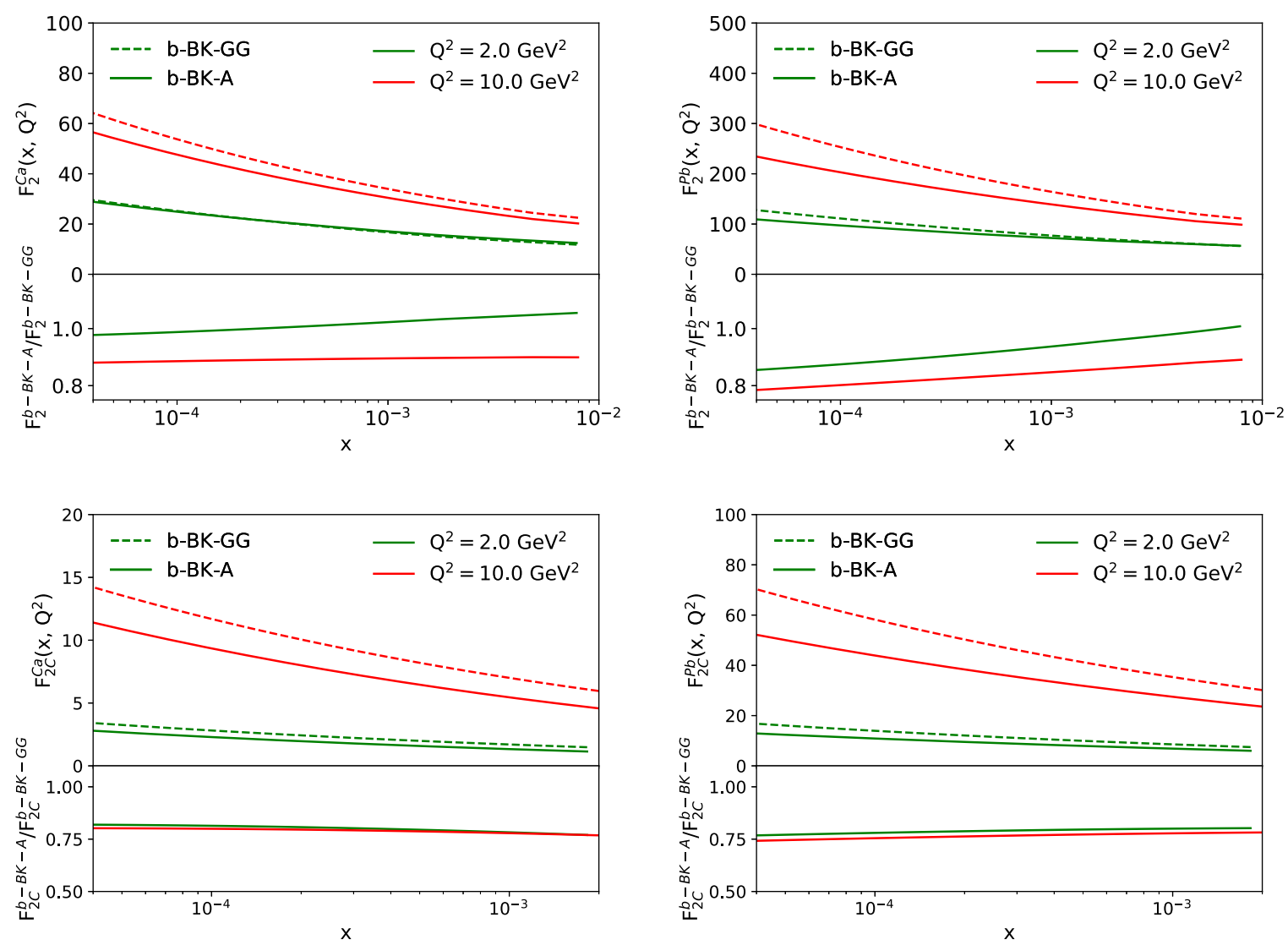

FIG. 5. The upper panels show the dependence on $x$ of the nuclear structure function $F_{2}^{A}\left(x, Q^{2}\right)$ computed in the b-BK-A and b-BK-GG approaches for two values of the photon virtuality $Q^{2}$ and two nuclei: $\mathrm{Ca}$ (left) and $\mathrm{Pb}$ (right). The ratio of the structure functions in the b-BK-A and b-BK-GG approaches is shown in the lower part of those panels. The bottom panels show the contribution of charm; that is, they show the structure function $F_{2 C}^{A}\left(x, Q^{2}\right)$.

in Ref. [13], the numerical results do not depend strongly on these choices.

\section{B. Predictions for the nuclear structure function}

The nuclear structure functions $F_{2}^{A}\left(x, Q^{2}\right)$ for $\mathrm{Ca}$ and $\mathrm{Pb}$ are shown in the upper panels of Fig. 5 as a function of $x$ for two values of the photon virtuality $Q^{2}$. Results in both approaches, b-BK-A and b-BK-GG, are shown in the figure, which also shows the ratio of both predicted structure functions. There is a clear difference between both sets of results. Furthermore, the difference shows a dependence on $x$, on $Q^{2}$, and a striking dependence on $A$, where the difference between both approaches grows from small to large nuclei. Note that the parameters of the model are quite constrained already: HERA data restricts the values to be taken for the proton target, the parameters of Woods-Saxon distributions are well know from sources independent to this analysis, and the initial condition is fixed by using the EPPS16 parametrization which concentrates our best knowledge of the nuclear structure at those scales. In this sense, one can consider the values of $F_{2}^{A}\left(x, Q^{2}\right)$ at larger rapidities as a true prediction with very little room to play with the parameter values.

A measurement of this structure function is expected to be one of the first results of any future EIC. Given the precision expected from these machines [27], these measurements will select which of the two approaches describes better the data. In our opinion, the b-BK-GG approach is not as consistent because, at small $x$ values, it is doubtful that the correct degrees of freedom are the nucleons. For example, there are studies within a Glauber approach, but with subnucleon degrees of freedom, that already point in this direction [37]. We think that, for saturation studies, the target should enter at the initial condition as one entity and this has to be evolved using the BK equation, which we call here the b-BK-A case. Data will have the answer.

The lower panels of Fig. 5 show that the difference between the b-BK-A and b-BK-GG approaches are larger for the charm structure function, $F_{2 C}^{A}\left(x, Q^{2}\right)$, than for the inclusive case, and that there is a very soft dependence on kinematic variables and nucleus species. A measurement of $F_{2 C}^{A}\left(x, Q^{2}\right)$ would offer additional stringent constraints to predictions of the structure function of nuclei.

\section{Predictions for the nuclear suppression factor}

As a final observable we present the nuclear suppression factor, defined as the ratio $R_{p A} \equiv F_{2}^{A}\left(x, Q^{2}\right) /\left[A F_{2}^{p}\left(x, Q^{2}\right)\right]$, which is expected to be unity if the structure of a free nucleon is equal to that of a bound nucleon. This ratio is the most direct 

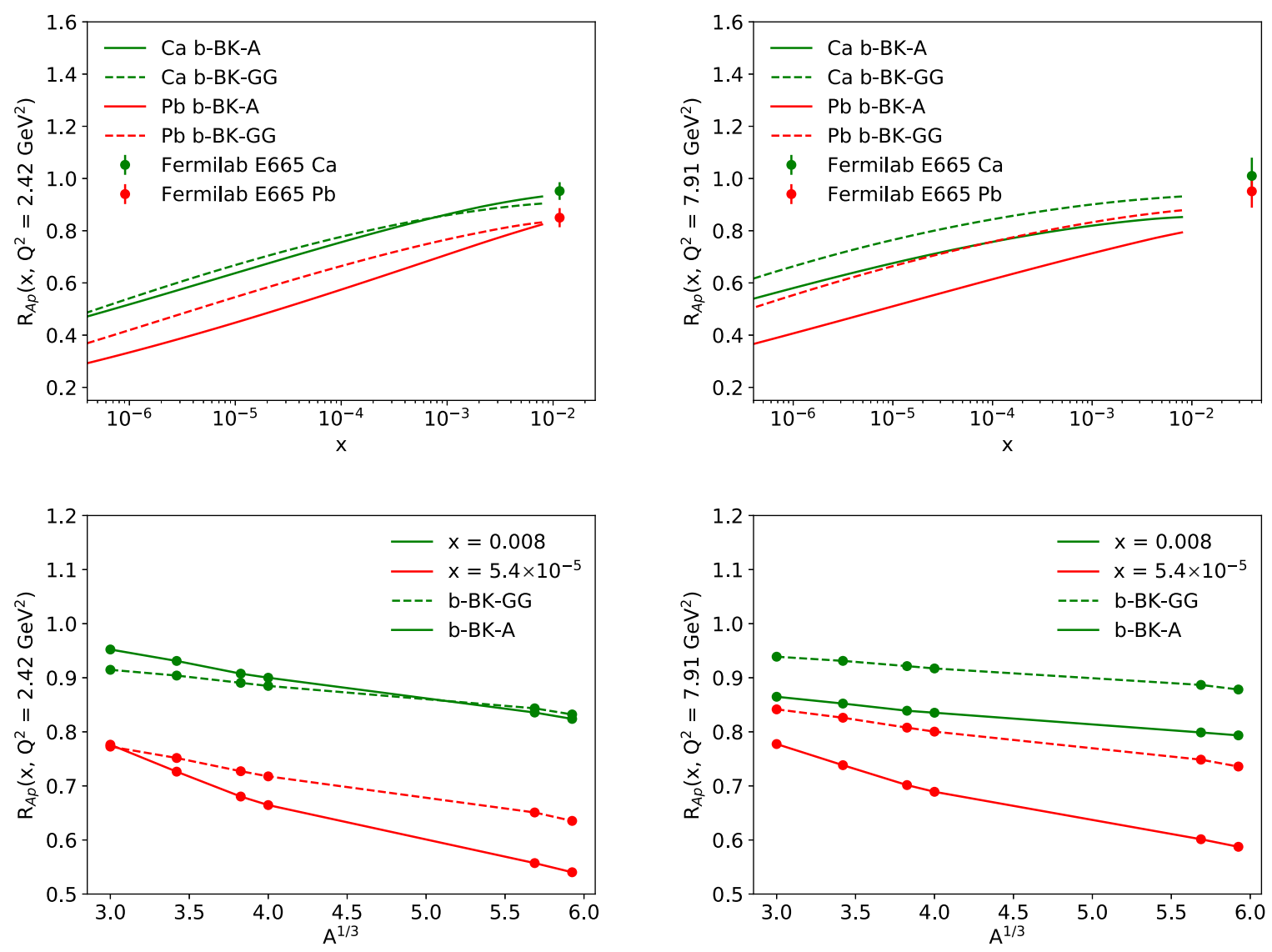

FIG. 6. Nuclear suppression factor at two values of the photon virtuality $Q^{2}=2.42 \mathrm{GeV}^{2}$ (left) and $Q^{2}=7.91 \mathrm{GeV}^{2}$ (right) as a function of $x$ for $\mathrm{Ca}$ and $\mathrm{Pb}$ (upper panels) and as a function of $A$ at different fixed values of $x$ (lower panels). The predictions are compared with data from Ref. [38].

way to observed nuclear shadowing, which for small $x$ is dominated by gluon shadowing and thus may be an important tool to determine the behavior of saturation across different nuclei.

This factor is shown in the upper panels of Fig. 6 as a function of $x$. Existing data at the same $Q^{2}$ from Ref. [38] is also shown as a cross-check of the procedure. For the $x$ dependence of $R_{p A}$ one sees a linear decrease (in logarithmic scale) towards small $x$ for both nuclei, but the linear behavior is reached later for the lighter nucleus, especially at higher $Q^{2}$ scales.

The b-BK-A computation predicts stronger shadowing than the b-BK-GG case, with this behavior seemingly dependent on $Q^{2}$. The same figure also shows, in the bottom panels, the $A$ dependence of the nuclear suppression factor for two $Q^{2}$ scales and for two values of $x$. As expected, shadowing becomes stronger as the size of the nucleus grows. The different behavior of shadowing for different nuclei in the b-BK-A and b-BK-GG is clearly seen in this observable. The difference in the nuclear modification factor between both approaches can be traced back to the different saturation scale shown in Fig. 4. Larger saturation scales mean that the wavefront of the dipole scattering amplitude reaches smaller values of $r$. This means that the integral in Eq. (14) yields a larger value and that $R_{p A}$ is closer to one.

Figure 7 shows the comparison of our predictions with those obtained using EPPS16, which is considered a standard of our current knowledge of nuclear shadowing. The comparison is done for $\mathrm{Ca}$ and $\mathrm{Pb}$ as middle and large nuclei. The predictions are compared with data from Ref. [38]. Note that the predictions are at a $Q^{2}$ scale of $2.42 \mathrm{GeV}^{2}$, which we considered the lowest we would like to go to stay in a somehow perturbative scale. But the data are measured at a different $Q^{2}$ for each $x$ value (as illustrated by the use of empty markers for data at smaller $Q^{2}$ ). The $Q^{2}$ values are reported in the figure.

Focusing on the predictions, the behavior at small $x$ is definitely different for the EPPS16 and BK computations. Note that the difference between EPPS16 and b-BK at the initial scale used for the BK evolution have two origins: one, that the parameter of the initial scale shown in Fig. 1 is chosen by comparing with larger values of $Q^{2}$ than those shown in Fig. 7, and two, that the prediction for the structure of the proton is substantially different for EPPS16 and for the b-BK approach reported in Refs. [19,20]. Given that the difference between the approaches goes beyond a normalization factor and shows a strong $x$ dependence, data from future EICs are expected to be precise enough to decide which prediction is closer to reality.

Comparing with the currently available data, and taking into account (i) the different $Q^{2}$ in data and predictions, and (ii) that for measurements the values are quite low (even below what one would expect to be valid for an approach based 

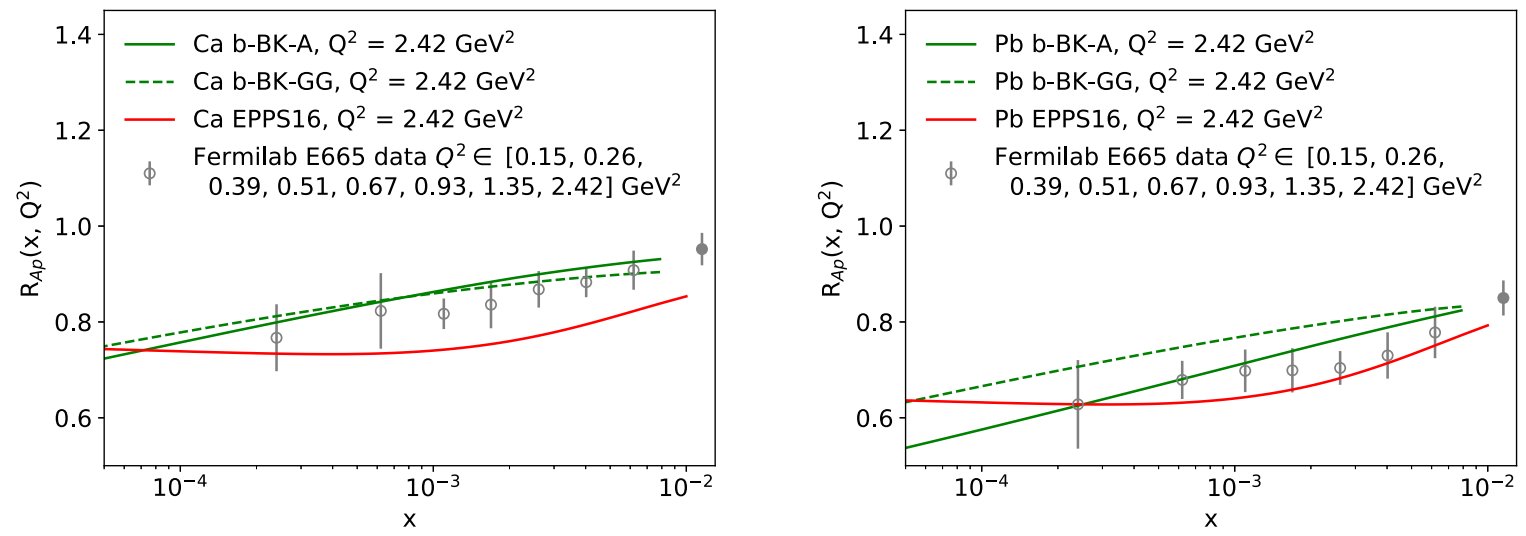

FIG. 7. Nuclear suppression factor for $\mathrm{Ca}$ (left) and $\mathrm{Pb}$ (right) for $Q^{2}=2.42 \mathrm{GeV}^{2}$. The data-points correspond to $Q^{2}$ values of $[0.15$, $0.26,0.39,0.51,0.67,0.93,1.35,2.42]$ from left to right. Only the data point represented by a solid marker correspond to the $Q^{2}$ used for the predictions. See text for details.

on perturbative $\mathrm{QCD}$ ), the $\mathrm{b}-\mathrm{BK}-\mathrm{A}$ prediction seems to do a reasonable job of describing data. The EPPS16 prediction also does quite well for $\mathrm{Pb}$, but slightly worse for $\mathrm{Ca}$. The b-BKGG prediction on the other hand is good when comparing with $\mathrm{Ca}$, but it suffers a bit when compared with $\mathrm{Pb}$.

\section{SUMMARY AND OUTLOOK}

The dipole scattering amplitudes, including the impactparameter dependence, for different nuclei have been obtained by solving the BK equation with the collinearly improved kernel. These amplitudes have been used to predict structure functions and nuclear saturation factors in kinematic ranges of interest for future EICs, such as those currently planned in the USA and at CERN. We followed two approaches: modeling the target directly as a nucleus and using Woods-Saxon parametrizations (denoted as b-BK-A above), and solving for a proton and using a Glauber-Gribov prescription to go to the nuclear level (denoted as b-BK-GG above). In our opinion, in the context of BK studies, the b-BK-A approach is more appropriate because it does not use the nucleons as degrees of freedom at small $x$. Furthermore, it avoids the exponentiation of the contribution at large impact parameters found in Eqs. (6) and (7).

We find sizable differences between these approaches. These differences show a dependence on $x, Q^{2}$, and $A$ such that data from a future EIC will be able to select the most appropriate approach for the description of data. We also compared nuclear suppression factors with those predicted by the EPPS16 formalism, which is taken as a standard of our current knowledge of nuclear shadowing. We find that all three approaches yield different predictions and that the b-BK-A computation seems to provide a better description of existing data.

These studies show that the data expected from a future EIC have the capability of selecting the best theoretical approach and thus to advance our understanding of the nuclear structure, of shadowing, and of the high-energy limit of QCD.

The dipole scattering amplitudes computed in this work are publicly available [39] along with macros and instructions to facilitate their use for anybody interested.

\section{ACKNOWLEDGMENTS}

This work was partially performed within the activities of the Centre of Advanced Applied Sciences with the number: CZ.02.1.01/0.0/0.0/16-019/0000778. The Centre of Advanced Applied Sciences is co-financed by the European Union. This work has also been partially supported by grant LTC17038 of the INTER-EXCELLENCE program at the Ministry of Education, Youth and Sports of the Czech Republic, the European Regional Development Fund-Project "Brookhaven National Laboratory - participation of the Czech Republic" (No. CZ.02.1.01/0.0/0.0/16013/0001569) and the COST Action CA15213 THOR. Computational resources were provided by the CESNET LM2015042 grant and the CERIT Scientific Cloud LM2015085, provided under the program Projects of Large Research, Development, and Innovations Infrastructures.
[1] A. Accardi et al., Eur. Phys. J. A 52, 268 (2016).

[2] J. Abelleira Fernandez et al. (LHeC Study Group), J. Phys. G 39, 075001 (2012).

[3] H. Abramowicz et al. (ZEUS, H1 Collaborations), Eur. Phys. J. C 75, 580 (2015).

[4] N. Armesto, J. Phys. G 32, R367 (2006).

[5] A. H. Mueller and J.-w. Qiu, Nucl. Phys. B 268, 427 (1986).

[6] N. N. Nikolaev and B. Zakharov, Z. Phys. C 49, 607 (1991).
[7] J. L. Albacete and C. Marquet, Prog. Part. Nucl. Phys. 76, 1 (2014).

[8] L. V. Gribov, E. M. Levin, and M. G. Ryskin, Phys. Rep. 100, 1 (1983).

[9] I. Balitsky, Nucl. Phys. B 463, 99 (1996).

[10] Y. V. Kovchegov, Phys. Rev. D 60, 034008 (1999).

[11] Y. V. Kovchegov and H. Weigert, Nucl. Phys. A 784, 188 (2007). 
[12] J. L. Albacete and Y. V. Kovchegov, Phys. Rev. D 75, 125021 (2007).

[13] E. Iancu, J. D. Madrigal, A. H. Mueller, G. Soyez, and D. N. Triantafyllopoulos, Phys. Lett. B 750, 643 (2015).

[14] E. Iancu, J. D. Madrigal, A. H. Mueller, G. Soyez, and D. N. Triantafyllopoulos, Phys. Lett. B 744, 293 (2015).

[15] J. L. Albacete, N. Armesto, J. G. Milhano, P. Quiroga-Arias, and C. A. Salgado, Eur. Phys. J. C 71, 1705 (2011).

[16] K. J. Golec-Biernat and A. Stasto, Nucl. Phys. B 668, 345 (2003).

[17] J. Berger and A. M. Staśto, Phys. Rev. D 83, 034015 (2011).

[18] J. Berger and A. M. Staśto, Phys. Rev. D 84, 094022 (2011).

[19] J. Cepila, J. G. Contreras, and M. Matas, Phys. Rev. D 99, 051502(R) (2019).

[20] D. Bendova, J. Cepila, J. G. Contreras, and M. Matas, Phys. Rev. D 100, 054015 (2019).

[21] B. Ducloué, E. Iancu, A. Mueller, G. Soyez, and D. Triantafyllopoulos, J. High Energy Phys. 04 (2019) 081.

[22] G. Beuf, H. Hänninen, T. Lappi, and H. Mäntysaari, arXiv:2007.01645 [hep-ph].

[23] N. Armesto, Eur. Phys. J. C 26, 35 (2002).

[24] E. R. Cazaroto, F. Carvalho, V. P. Goncalves, and F. S. Navarra, Phys. Lett. B 671, 233 (2009).

[25] L. Agozzino, P. Castorina, and P. Colangelo, Eur. Phys. J. C 74, 2828 (2014).
[26] C. Marquet, M. R. Moldes, and P. Zurita, Phys. Lett. B 772, 607 (2017).

[27] E. C. Aschenauer, S. Fazio, M. A. C. Lamont, H. Paukkunen, and P. Zurita, Phys. Rev. D 96, 114005 (2017).

[28] A. Sabio Vera, Nucl. Phys. B 722, 65 (2005).

[29] I. Balitsky and G. A. Chirilli, Phys. Rev. D 77, 014019 (2008).

[30] C. Loizides, J. Kamin, and D. d'Enterria, Phys. Rev. C 97, 054910 (2018); 99, 019901(E) (2019).

[31] H. De Vries, C. De Jager, and C. De Vries, At. Data Nucl. Data Tables 36, 495 (1987).

[32] K. J. Eskola, P. Paakkinen, H. Paukkunen, and C. A. Salgado, Eur. Phys. J. C 77, 163 (2017).

[33] A. Buckley, J. Ferrando, S. Lloyd, K. Nordström, B. Page, M. Rüfenacht, M. Schönherr, and G. Watt, Eur. Phys. J. C 75, 132 (2015).

[34] V. Bertone, S. Carrazza, and J. Rojo, Comput. Phys. Commun. 185, 1647 (2014).

[35] S. Carrazza, A. Ferrara, D. Palazzo, and J. Rojo, J. Phys. G 42, 057001 (2015).

[36] K. J. Golec-Biernat and M. Wusthoff, Phys. Rev. D 59, 014017 (1998).

[37] C. Loizides, Phys. Rev. C 94, 024914 (2016).

[38] M. R. Adams et al. (E665 Collaboration), Z. Phys. C 67, 403 (1995).

[39] See website https://hep.fjfi.cvut.cz/. 